\title{
Effects of polyvinylpyrrolidone-iodine on tendon-bone healing in a rabbit extra-articular model
}

\author{
PENG ZHANG $^{1 *}$, YUNLONG ZHI ${ }^{1 *}$, HONGWEI FANG $^{2}$, ZIYING WU $^{1}$, \\ TIANWU CHEN $^{1}$, JIA JIANG ${ }^{1}$ and SHIYI CHEN ${ }^{1}$ \\ ${ }^{1}$ Department of Sports Medicine, Huashan Hospital, Fudan University, Shanghai 200040; \\ ${ }^{2}$ Department of Anesthesiology, Bengbu Medical College, Bengbu, Anhui 233004, P.R. China
}

Received January 25, 2016; Accepted February 17, 2017

DOI: $10.3892 / \mathrm{etm} .2017 .4359$

\begin{abstract}
Polyvinylpyrrolidone-iodine (PVP-I) is a broad-spectrum antimicrobial agent, but its effects on tendon-bone healing are unclear. The purpose of this study was to investigate the effects of PVP-I on bone marrow mesenchymal stem cells (BMSCs) in vitro and on tendon-bone healing in vivo. In this study, following investigation of the concentration-dependent effects of PVP-I on the viability and osteogenic differentiation of BMSCs, the appropriate concentration of PVP-I was selected for animal experiments. New Zealand white rabbits received autologous tendon transplantation with and without PVP-I treatment of the graft tendon. Subsequently, histological examination, biomechanical testing and reverse transcription-quantitative polymerase chain reaction (RT-qPCR) analyses were conducted. At 6 weeks post-surgery, connective tissue and osteogenesis was observed at the tendon-bone interface in the PVP-I group. At 12 weeks post-surgery, the interface width in the PVP-I group was much narrower compared with that of the control group. Furthermore, the biomechanical properties of the PVP-I group were significantly stronger than those in the control group $(\mathrm{P}<0.05)$. RT-qPCR examination revealed that the mRNA levels of bone morphogenetic protein-2 and osteopontin in the PVP-I group were higher than those in the control group at 6 weeks $(\mathrm{P}<0.05)$. In conclusion, these results indicated that PVP-I promoted tendon-bone healing via osteogenesis.
\end{abstract}

Correspondence to: Professor Shiyi Chen, Department of Sports Medicine, Huashan Hospital, Fudan University, 12 Middle Wulumuqi Road, Shanghai 200040, P.R. China

E-mail: chenshiyifudan@sina.com

*Contributed equally

Key words: polyvinylpyrrolidone-iodine, bone marrow mesenchymal stem cells, osteogenesis, anterior cruciate ligament, tendon-bone healing

\section{Introduction}

Rupture of the anterior cruciate ligament (ACL) is a common and devastating knee injuries. The aim of surgery is to restore knee functional stability, eliminate symptoms and prevent degenerative joint changes, particularly for young individuals and athletes. Hamstring tendons remain a popular choice for autograft in ACL reconstruction $(1,2)$. Furthermore, numerous studies have elucidated that it is possible to achieve successful ACL reconstruction with a hamstring autograft (3-8). This is because hamstring tendons have certain advantages, including low donor-site morbidity, few kneeling problems, good extension strength and a low incidence of long-term degenerative joint disease. However, the long healing time of soft tissue tendon grafts within the bone tunnels remains unresolved. Previously, studies have focused on strategies to augment tendon-bone healing by modulating the biological or the biomechanical environment, or both (9-12).

Polyvinylpyrrolidone-iodine (PVP-I) is a broad-spectrum antiseptic introduced in 1956 (13). PVP-I irrigation is commonly used to reduce the risk of infection in surgery. It has a non-selective mechanism of action, and a high concentration of PVP-I is potentially toxic to both infectious and host cells. However, the biological effects of low-concentration PVP-I on joint tissue cells are largely unclear. There is considerable debate about the biocompatibility of PVP-I. In vitro study has indicated that the dilute solutions of PVP-I that are used to sterilize open wounds could cause fibroblast growth inhibition (14). In another study, it was reported that even when diluted to a $0.35 \%$ concentration, PVP-I continued to have a pronounced chondrotoxic effect on bovine articular cartilage (15).

Schmidlin et al (16) demonstrated that when briefly exposed to PVP-I for $10 \mathrm{sec}$, osteoblasts manifested more mineral deposition by Alizarin red staining compared with osteoblasts that were not exposed to it. Jiang et al (17) reported that PVP-I enhanced the osteogenic differentiation of bone marrow mesenchymal stem cells (BMSCs) and osteoblasts, and they considered that the function was through accumulation of the extracellular matrix (ECM). Furthermore, they indicated that PVP-I had the potential to repair osteochondral defects. The effects of PVP-I on the tendon-bone healing that 
is crucial in ACL reconstruction have not yet been investigated. In the present study, PVP-I was applied in order to treat an autologous tendon in a rabbit extra-articular model and to investigate the effects of PVP-I on tendon-bone healing.

\section{Materials and methods}

Cell isolation and culture. BMSCs were extracted and isolated from tibia and femur bone marrow of a single 1-week old male New Zealand white rabbit. The rabbit was obtained from the Department of Laboratory Animal Science of Fudan University (Shanghai, China). The environment in which the rabbit was kept was as follows: Temperature, $22^{\circ} \mathrm{C}$; relative humidity, $50 \%$; 12-h light/dark cycle; and access to food and water ad libitum. In brief, $2 \mathrm{ml}$ bone marrow was obtained, mixed with 2 ml low-glucose Dulbecco's modified Eagle's medium (DMEM; Thermo Fisher Scientific, Inc., Waltham, MA, USA) and centrifuged at $1,200 \times g$ for $5 \mathrm{~min}$. The supernatant was then removed and the remnant cells were suspended and cultured in low-glucose DMEM supplemented with $10 \%$ fetal bovine serum (FBS; Thermo Fisher Scientific, Inc.) and $1 \%$ penicillin-streptomycin. Cultures were incubated at $37^{\circ} \mathrm{C}$ and $5 \% \mathrm{CO}_{2}$. Furthermore, the medium was exchanged every 3 days, and the adherent cells were defined as BMSCs. When reaching 80-90\% confluence, adherent cells were released from the flask with $0.05 \%$ trypsin and subcultured. In addition, BMSCs from the second to fifth passages were harvested and used for subsequent experiments.

Cell viability assay. Cell viability was quantified via the MTT dye reduction assay. BMSCs $\left(1 \times 10^{5}\right.$ cells/well) were seeded in 24-well plates with serum-free low-glucose DMEM containing different concentrations of PVP-I (1, 10, 50, 100, 200, 500 and 1,000 $\mu \mathrm{M}$; Shanghai Bangshili Disinfectant Co. Ltd., Shanghai, China) and cultured for $24 \mathrm{~h}$. There were 3 wells for each concentration. After $24 \mathrm{~h}$, MTT reagent (5 mg/ml; Sigma-Aldrich, Inc.; Merck KGaA, Darmstadt, Germany) was added to each well and incubated for $4 \mathrm{~h}$ in $5 \%$ $\mathrm{CO}_{2}$ at $37^{\circ} \mathrm{C}$. Next, the medium was removed and formazan salts were dissolved in dimethyl sulfoxide (Sigma-Aldrich, Inc.; Merck KGaA). The absorbance was then determined at $540 \mathrm{~nm}$ using a spectrophotometer (Epoch; Biotek, Winooski, VT, USA).

Alkaline phosphatase (ALP) activity of BMSCs. Osteogenesis can be demonstrated by the expression of ALP. BMSCs $\left(1 \times 10^{5}\right.$ cells/well) were seeded in 24-well plates in low-glucose DMEM with $10 \%$ FBS and $1 \%$ penicillin-streptomycin alone or with various concentrations of PVP-I (10, 50 and $100 \mu \mathrm{M})$. After culture for 7 or 14 days, the osteogenic differentiation of the BMSCs was evaluated by ALP activity assays using an ALP kit according to the manufacturer's protocol (A059-2; Nanjing Jiancheng Bioengineering Institute, Nanjing, China). This experiment was repeated 3 times.

Mineralization of BMSCs. Alizarin red is a dye that selectively binds to calcium salts and is widely used to stain calcium deposits, which are indicators of mature osteocytes. Alizarin red staining was used to detect mineralization (18). The cells were cultured with low-glucose DMEM with $10 \%$ FBS and
$1 \%$ penicillin-streptomycin for 3 days, and the medium was replaced with osteogenic induction medium (OIM) for another 14 days. The OIM consisted of $10 \mathrm{mM} \beta$-glycerol phosphate, $0.1 \mu \mathrm{M}$ dexamethasone and $50 \mathrm{mg} / \mathrm{ml}$ ascorbic acid (all from Sigma-Aldrich, Inc.; Merck KGaA) in high-glucose DMEM containing $10 \%$ FBS and $1 \%$ penicillin-streptomycin. Furthermore, different concentrations of PVP-I (10, 50 and $100 \mu \mathrm{M})$ were added to the OIM.

After culture for 14 days, the cells were washed 3 times with phosphate-buffered saline and then fixed in $2.5 \%$ glutaraldehyde solution for $10 \mathrm{~min}$. Next, the cells were washed with double-distilled water and stained with Alizarin red solution for $5 \mathrm{~min}$. Following staining, double-distilled water was used to wash off the residual staining solution and the cells were dried at room temperature.

Animal study design and surgical procedure. In total, 48 adult male New Zealand white rabbits (age, 12 weeks; weight, $2.5 \pm 0.3 \mathrm{~kg}$ ) were subjected to an extra-articular tendon-bone healing surgery. The environment in which the rabbits were kept was as follows: Temperature, $22^{\circ} \mathrm{C}$; relative humidity, 50\%; 12-h light/dark cycle; and access to food and water ad libitum. The animals were anaesthetized by administration of an intravenous injection of $3 \%$ pentobarbital $(30 \mathrm{mg} / \mathrm{kg})$. In a supine position, the bilateral lower limbs of rabbits were disinfected. An incision was made along the right Achilles tendon and partial-thickness of the Achilles tendon was harvested as a graft. In the experimental group, the Achilles tendon was immersed in $100 \mu \mathrm{M}$ PVP-I for $30 \mathrm{~min}$, while in the control they were immersed in normal saline for $30 \mathrm{~min}$ instead. A 2.5-mm-diameter drill was used to create the bone tunnels in the proximal tibia in the direction of $60^{\circ}$ to the longitudinal axis of the tibia as described previously (19). The prepared graft was then passed through the bone tunnel manually and the two ends of the grafts were sutured to the adjacent soft tissue with 3-0 Ethibond sutures (Johnson \& Johnson, New Brunswick, NJ, USA), while 5-mm-long graft was kept outside the bone tunnel for future biomechanical experiments. Next, the wound was closed layer by layer. Following the surgery, the rabbits were injected with penicillin (40 kU/kg) for 3 days. The rabbits were then kept in the cages for free activities following a surgical procedure. After 6 and 12 weeks, the rabbits were sacrificed in order to harvest tissue specimens for histological examination, biomechanical testing and reverse transcription-quantitative polymerase chain reaction (RT-qPCR) analysis. The animal experiments were approved by the Animal Care and Use Committee of Shanghai Medical College of Fudan University.

Histological examination. Immediately after sacrifice, the specimens were fixed in $10 \%$ neutral-buffered formalin for $48 \mathrm{~h}$, decalcified in formic acid, dehydrated through an alcohol gradient, cleared and then embedded in paraffin wax. The samples were cut into $5-\mu \mathrm{m}$ slides perpendicular to the longitudinal axis of the bone tunnel. These sections were then stained with hematoxylin and eosin for histological evaluation. The graft-bone interface was visualized by inverted-light microscopy (Leica DM6000B; Leica Microsystems GmbH, Wetzlar, Germany), and digital images were captured using DP Manager (Leica DM6000B; Leica Microsystems GmbH). 
Mechanical evaluation. A total of six fresh specimens from each group were prepared for mechanical testing. The peripheral tissues, except for the tendon graft, were carefully excised from the graft-tibia complex. The mechanical testing was performed using an electronic universal materials testing system machine (AGS-X; Shimadzu, Co., Kyoto, Japan). Prior to the tensile test, the samples were preloaded with a static preload of $1 \mathrm{~N}$ for $5 \mathrm{~min}$. Following the preloading, the ultimate failure load $(\mathrm{N})$ was determined immediately with an elongation rate of $2 \mathrm{~mm} / \mathrm{min}$. Furthermore, the load-deformation curve was recorded, from which $\mathrm{N}$ was measured. Stiffness $(\mathrm{N} / \mathrm{mm})$ was calculated from the slope of the linear region of the load-deformation curve at the maximal load-to-failure point. For each sample, the test was completed when the graft was ruptured or pulled out of the bone tunnel.

$R T-q P C R$ analysis. The graft-tibia complex ( $\mathrm{n}=3$ limbs in each group at 6 or 12 weeks postoperatively) was harvested after sacrifice for RT-qPCR analysis. The total RNA from interfacial samples between host bone tunnel and graft were extracted using TRIzol reagent (Invitrogen; Thermo Fisher Scientific, Inc., Waltham, MA, USA) based on the manufacturer's instructions. The cDNA was generated using reverse-transcriptase M-MLV (D2640A; Takara Bio, Inc., Otsu, Japan) according to the manufacturer's protocol. qPCR was performed with SYBR Premix Ex Taq (DRR041A; Takara Bio, Inc.), and then detected using a qPCR system (TP800; Takara Bio, Inc.).

The primers for qPCR were as follows: $\beta$-actin, forward, 5'-CCAAGGCCAACCGCGAGAAGATGA-3' and reverse, 5'-GCAGCGCGTAGCCCTCGTAGATGG-3'; bone morphogenetic protein-2 (BMP-2), forward, 5'-GGAATGACTGGA TTGTGGCT-3' and reverse, 5'-TGAGTTCTGTCGGGA CACAG-3'; and osteopontin (OPN), forward, 5'-GTGGAC AGCGAGGACTTGGATG-3' and reverse, 5'-GGCCTC GCGCTTATATTGTCTGG-3'. The PCR cycling conditions were as follows: $95^{\circ} \mathrm{C}$ for $2 \mathrm{~min}$, followed by 40 cycles of $95^{\circ} \mathrm{C}$ for $15 \mathrm{sec}$ and $60^{\circ} \mathrm{C}$ for $30 \mathrm{sec}$. Relative expression levels were normalized to $\beta$-actin, according to the $2^{-\Delta \Delta \mathrm{Cq}}$ method (20).

Statistical analysis. Data are presented as the mean \pm standard deviation. Student's t-test was performed to assess statistically significant differences in the results of different experimental groups. Statistical analysis was performed using SPSS Statistics 19.0 software package (IBM SPSS, Armonk, NY, USA). $\mathrm{P}<0.05$ was considered to indicate a statistically significant difference.

\section{Results}

BMSCs. Rabbit BMSCs grew well in the low-glucose medium. The cells proliferated to $80-90 \%$ confluence and were subcultured for 4-5 days before they were observed using optical microscopy at the third passage. The BMSCs demonstrated spindle-like morphology (Fig. 1).

Effects of different concentrations of PVP-I on BMSC viability. The cytotoxicity of PVP-I on BMSCs was initially tested to identify an appropriate concentration for further experiments. A serial concentration range of PVP-I (1, 10, 50, 100, 200, 500 and $1,000 \mu \mathrm{M}$ ) was added to the serum-free low-glucose DMEM, and cell viability was assessed by MTT assay. MTT assays demonstrated that PVP-I began to be detrimental to BMSCs at a concentration of $200 \mu \mathrm{M}$, and BMSC proliferation was clearly inhibited when the concentration of PVP-I in the medium reached $>500 \mu \mathrm{M}$. When the concentration of PVP-I was $1,000 \mu \mathrm{M}$, the majority of BMSCs did not survive after $24 \mathrm{~h}$. PVP-I $\leq 100 \mu \mathrm{M}$ had negligible toxic effects on BMSC proliferation when compared with the control group (Fig. 2). Therefore, the concentration of $100 \mu \mathrm{M}$ PVP-I was selected for further experiments.

Effects of PVP-I on osteogenesis of BMSCs. The ALP content was evaluated to directly reflect the osteogenesis of BMSCs. It was observed that the ALP expression of all groups varied with culture duration (Fig. 3). ALP activities increased markedly at day 14 when compared with those at day 7. After 7 and 14 days of culture, the ALP activities of the $100 \mu \mathrm{M}$ PVP-I groups were higher than that of the respective control group $(\mathrm{P}<0.05)$. When cultured in OIM, all groups had mineralized calcium deposits after osteogenic induction for 14 days as shown by Alizarin red staining, and there were no notable differences among the four groups (Fig. 4).

Histological examination. At 6 weeks after surgery, thick fibrous scar tissue had formed at the tendon-bone interface in the experimental and control groups. In the PVP-I group, connective tissue and osteogenesis was observed in the tendon-bone interface. At 12 weeks after surgery, fibrous connective tissue at the tendon-bone interface was narrow and indistinct in both groups. Furthermore, compared with the control group, the interface was narrower and even non-existent in some places, and less fibrous tissue formation was observed in the PVP-I group (Fig. 5).

Mechanical observations. Fixation of the tendon graft on the universal testing machine is shown in Fig. 6A and B. All grafts were pulled out from the bone tunnels and none were ruptured during the mechanical tests. At 6 weeks after surgery, there was no statistically significant difference in the load to failure between the PVP-I and control groups $(37.3 \pm 5.32$ vs. $33.1 \pm 2.90 \mathrm{~N}, \mathrm{P}=0.109$ ). At 12 weeks after surgery, the mean load to failure of the PVP-I group was higher than that of the control group (62.1 \pm 4.60 vs. $47.8 \pm 5.08 \mathrm{~N}, \mathrm{P}<0.001$; Fig. 6C). Furthermore, there was no significant difference in stiffness between the PVP-I and control groups at 6 weeks after surgery $(14.7 \pm 0.31$ vs. $14.4 \pm 0.60 \mathrm{~N} / \mathrm{mm}, \mathrm{P}=0.311)$. At 12 weeks after surgery, the average stiffness in the PVP-I group was significantly greater than that in the control group $(21.9 \pm 1.17$ vs. $15.4 \pm 1.39 \mathrm{~N} / \mathrm{mm}, \mathrm{P}<0.001$; Fig. $6 \mathrm{D}$ ).

$R T$ - $q P C R$. At week 6 , the PVP-I group showed significantly higher BMP-2 and OPN gene expression than the control group $(0.59 \pm 0.02$ vs. $0.27 \pm 0.04$ for $\mathrm{BMP}-2, \mathrm{P}<0.001$; $0.18 \pm 0.04$ vs. $0.13 \pm 0.03$ for $\mathrm{OPN}, \mathrm{P}<0.05)$. However, there were no significant differences in the mRNA levels of BMP-2 and OPN between the PVP-I and control groups $(0.29 \pm 0.04$ 


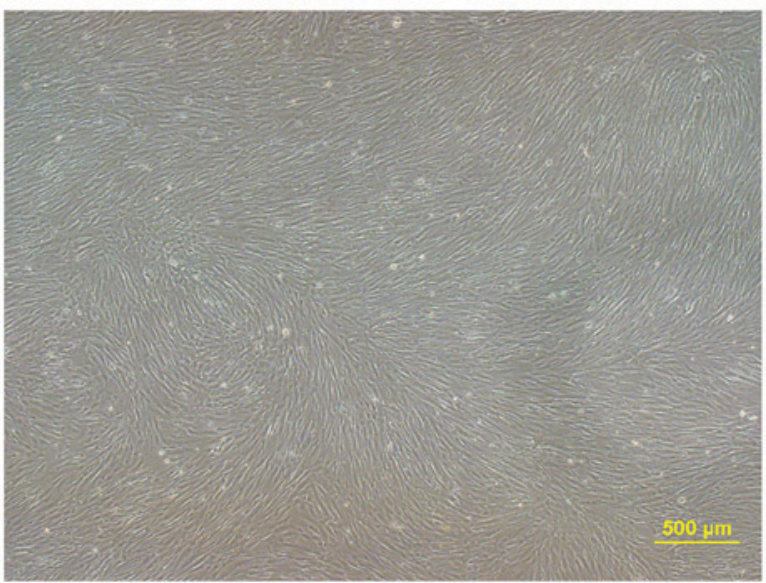

Figure 1. Morphology of rabbit bone marrow mesenchymal stem cells observed by optical microscopy. Scale bar, $500 \mu \mathrm{m}$.

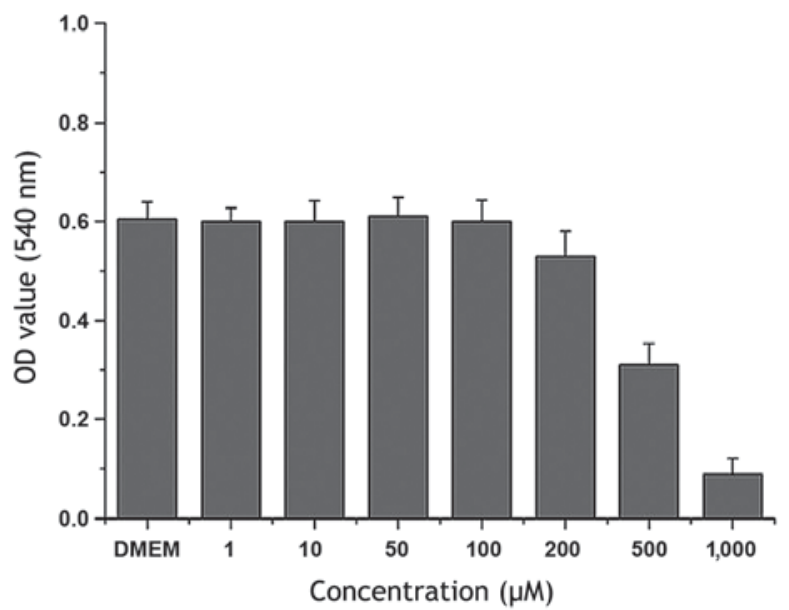

Figure 2. Bone marrow mesenchymal stem cell viability was assessed by MTT assay after $24 \mathrm{~h}$ of exposure to low-glucose DMEM supplemented with 1, 10, 50, 100, 200, 500 and 1,000 $\mu$ M PVP-I. DMEM represents the PVP-I-free control group. DMEM, Dulbecco's modified Eagle's medium; PVP-I, polyvinylpyrrolidone-iodine.

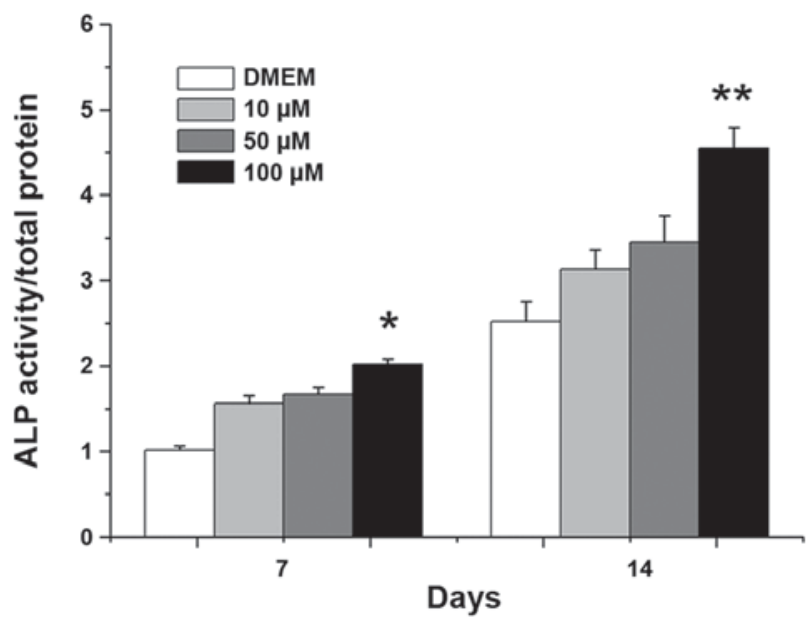

Figure 3. Relative activities of ALP for bone marrow mesenchymal stem cells cultured with different concentrations of PVP-I for 7 and 14 days. DMEM represents the PVP-I-free control group. ${ }^{~} \mathrm{P}<0.05$ vs. control group; ${ }^{* *} \mathrm{P}<0.001$ vs. control group. ALP, alkaline phosphatase; PVP-I, polyvinylpyrrolidone-iodine; DMEM, Dulbecco's modified Eagle's medium.
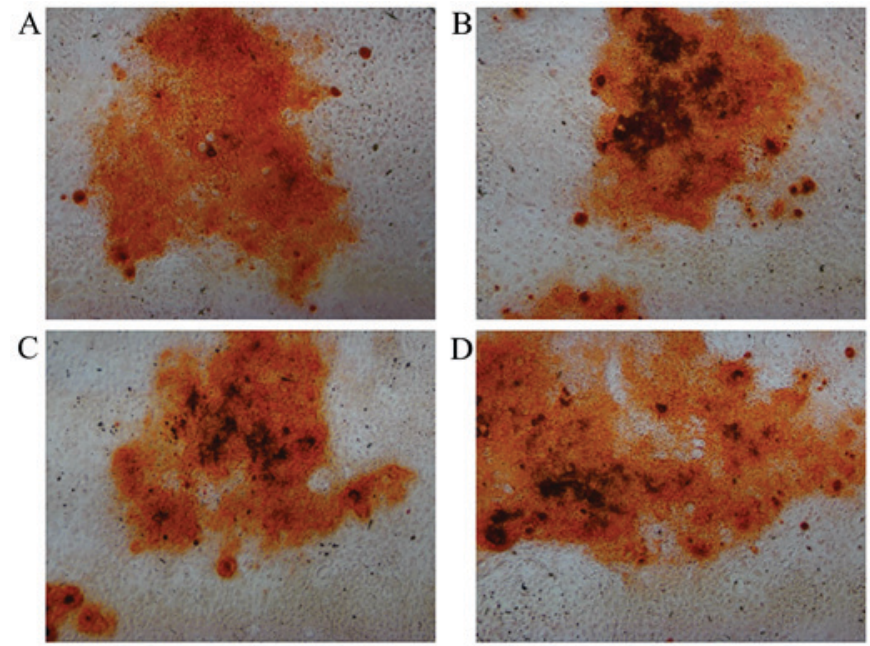

Figure 4. Alizarin red staining for bone marrow mesenchymal stem cells cultured with different concentrations of PVP-I for 14 days. Culture medium with (A) OIM, (B) OIM $+10 \mu \mathrm{M}$ PVP-I, (C) OIM $+50 \mu \mathrm{M}$ PVP-I and (D) OIM $+100 \mu \mathrm{M}$ PVP-I (magnification, x100). PVP-I, polyvinylpyrrolidone-iodine; OIM, osteogenic induction medium.
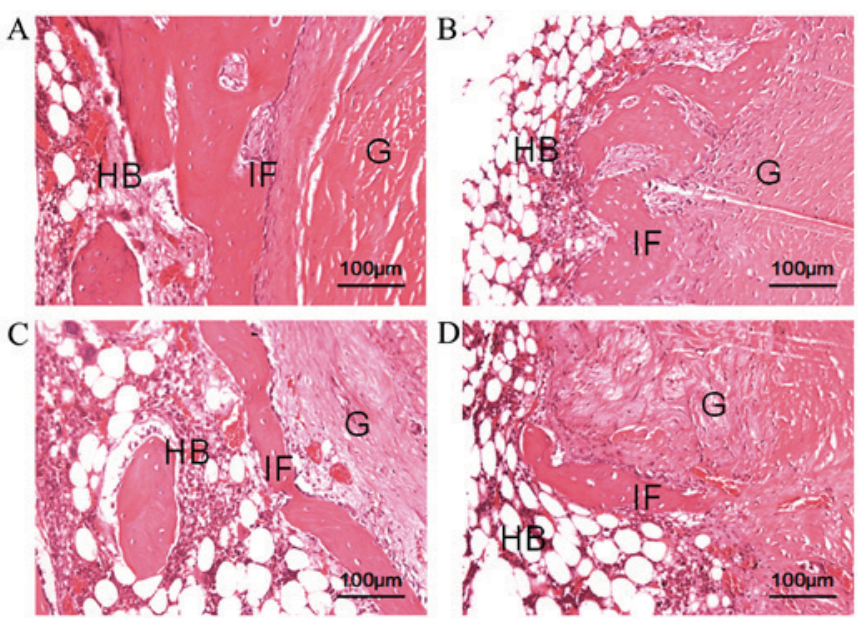

Figure 5. Histological analysis of the tendon-bone interface at 6 and 12 weeks after surgery (hematoxylin \& eosin staining). Images at 6 weeks in the (A) control and (B) PVP-I groups, and at 12 weeks in the (C) control and (D) PVP-I groups. Scale bar, $100 \mu \mathrm{m}$. HB, host bone; G, tendon graft; IF, interface; PVP-I, polyvinylpyrrolidone-iodine.

vs. $0.27 \pm 0.04$ for $\mathrm{BMP}-2, \mathrm{P}=0.098 ; 0.11 \pm 0.01$ vs. $0.09 \pm 0.01$ for OPN; $\mathrm{P}=0.063)$ at 12 weeks after surgery. In addition, the mRNA levels of BMP-2 and OPN decreased with time (Fig. 7).

\section{Discussion}

PVP-I is a commonly used antiseptic with a broad antimicrobial range. PVP-I lavation and irrigation significantly decrease the risk for surgical site infection, and are simple and economical to use (21-23). In addition to its antiseptic property, PVP-I has been demonstrated to promote bone healing $(16,17)$. To the best of our knowledge, the present study is the first to demonstrate that PVP-I is able to promote tendon-bone healing in vivo.

The results of the MTT experiments demonstrated that PVP-I $\leq 100 \mu \mathrm{M}$ had no clear inhibitory effect on BMSC 

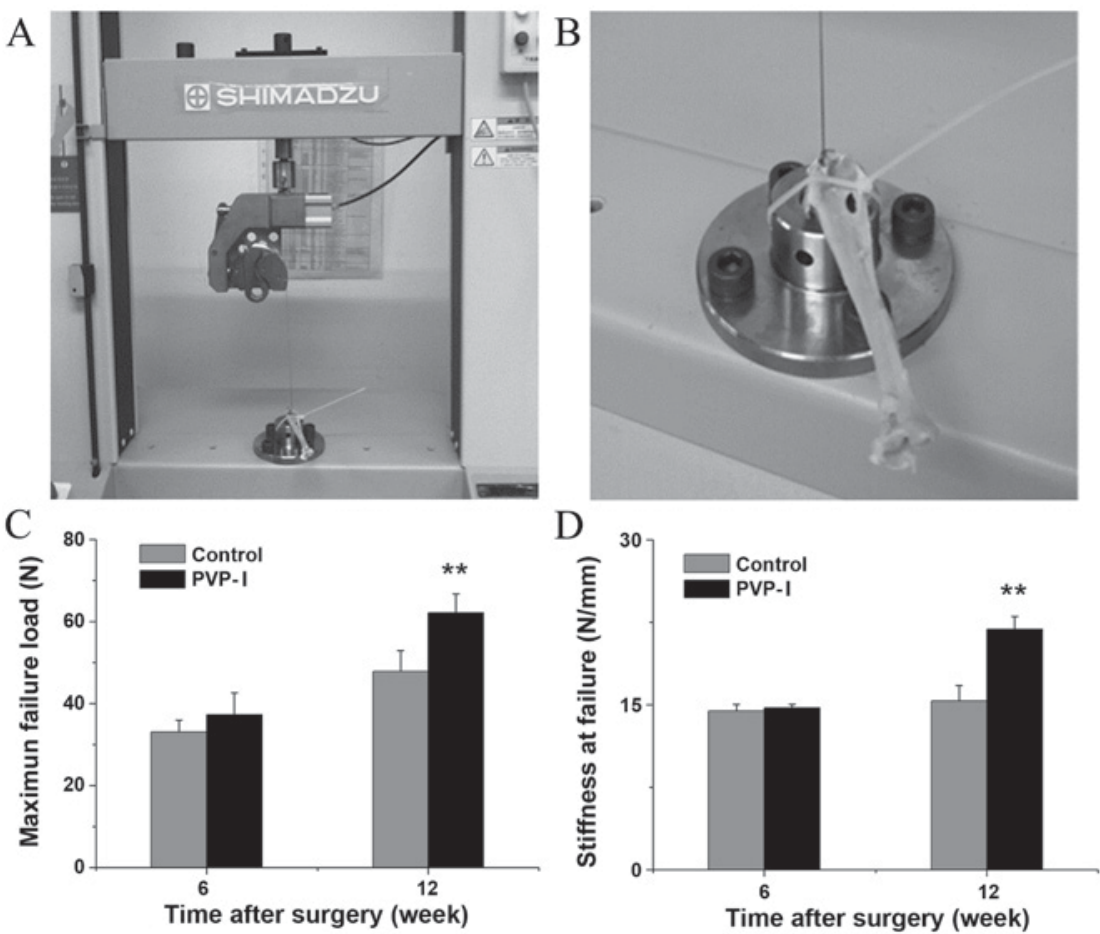

Figure 6. Mechanical tests for tendon-bone healing in a rabbit model at each time point after surgery. (A and B) Digital camera images showing fixation of the tendon graft on the universal testing machine. Comparison of (C) maximal failure load and (D) stiffness at failure between the control and PVP-I groups. ${ }^{* *} \mathrm{P}<0.001$ vs. control group. PVP-I, polyvinylpyrrolidone-iodine.

A

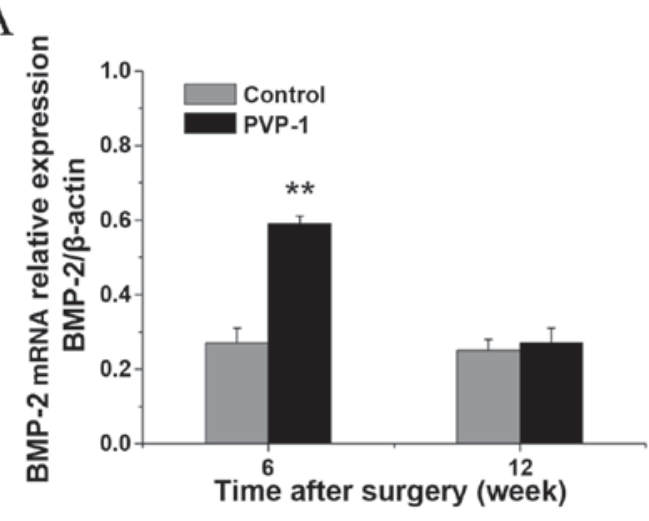

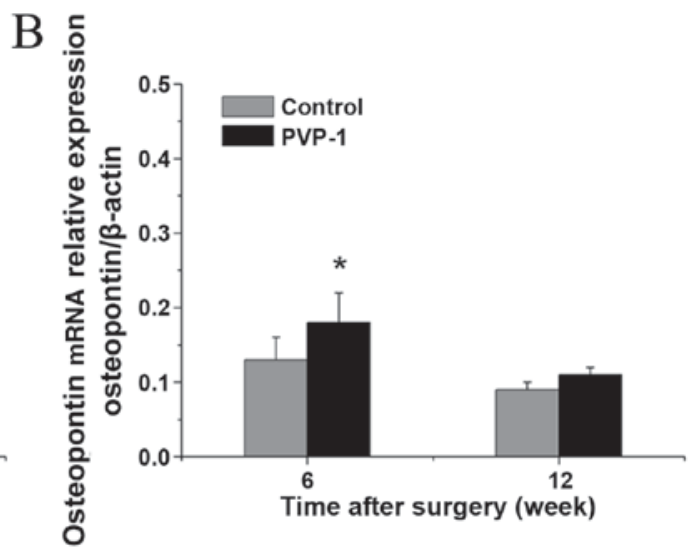

Figure 7. mRNA levels of (A) BMP-2 and (B) osteopontin at 6 and 12 weeks after surgery as determined by reverse transcription-quantitative polymerase chain reaction. ${ }^{*} \mathrm{P}<0.05$ vs. control group; ${ }^{* *} \mathrm{P}<0.001$ vs. control group. BMP-2, bone morphogenetic protein-2; PVP-1, polyvinylpyrrolidone-iodine.

proliferation. However, the cell growth began to be arrested when the PVP-I concentration was $>100 \mu \mathrm{M}$. Furthermore, cell growth was markedly inhibited when the concentration of PVP-I reached $500 \mu \mathrm{M}$. It has been reported that PVP-I exhibits toxicity to normal chondrocytes even at concentrations as low as $0.35 \%$ and after short exposure times (15). However, the molar concentration of PVP-I was not reported, making direct comparison with the results of the present study difficult. The differentiation of BMSCs was investigated in the present study through detection of endogenous ALP enzyme activity and ECM mineralization. At $100 \mu \mathrm{M}$, PVP-I exhibited a more evident osteoinductive effect compared with other groups with lower concentrations. Therefore, $100 \mu \mathrm{M}$ of PVP-I was selected as the optimum concentration for further animal experiments.
Previous studies have demonstrated that osteoinductive agents promote tendon-bone healing, and thus further enhance mechanical properties at the tendon-bone interface $(24,25)$. The present study found that, histologically, PVP-I promoted bone ingrowth into the tendon-bone interface at 12 weeks after surgery. Furthermore, no osteogenesis was evident in the control group. Fibrocartilage is a component in normal ACL insertion, but it was not evident in the present study in the PVP-I group, while new bone protruded into the interface. Maybe this was due to PVP-I having mainly osteogenic and few chondrogenic effects.

In the present study, it was shown that PVP-I significantly increased BMP-2 and OPN mRNA levels. Furthermore, the mRNA levels of BMP-2 in the PVP-I group were higher than those in the control group at 6 weeks after surgery. BMP-2 
belongs to the transforming growth factor-b superfamily, which is the most important cytokine in bone tissue engineering (26). In addition, BMP-2 may be specifically involved in the remodeling process of the bone, leading to osseous integration.

OPN is a multifunctional ECM component, which stimulates cell-cell adhesion, increases cell-ECM communication, promotes cell migration, alters intracellular calcium levels, and promotes calcium phosphate deposition (27). Previous research revealed that OPN levels were upregulated in the first few weeks after long bone fractures (28). Furthermore, OPN is considered an important factor in bone remodeling. The results of the present study indicated that a low-concentration of PVP-I increased the expression of BMP-2 and OPN, which could promote the osteogenic differentiation of BMSCs and enhance the formation of new bone.

However, there are some limitations in the present study. Firstly, only a single concentration of PVP-I was applied in small animals; therefore, the optimum concentration in tendon-bone healing was not identified. In future studies, different concentrations of PVP-I should be investigated, particularly in large animals, in order to search for the optimum concentration of PVP-I in tendon-bone healing. In conclusion, the results of the present study demonstrated that an appropriate concentration of PVP-I promoted tendon-bone healing via osteogenesis. This convenient and economical method may become a novel strategy for the promotion of tendon-bone healing in $\mathrm{ACL}$ reconstruction.

\section{Acknowledgements}

The present study was supported by the National $863 \mathrm{Hi}$-tech Project (grant no. 2015AA033703) and the National Natural Science Foundation of China (grant nos. 81271958, 81572108 and 81370052).

\section{References}

1. Magnussen RA, Granan LP, Dunn WR, Amendola A, Andrish JT, Brophy R, Carey JL, Flanigan D, Huston LJ, Jones M, et al: Cross-cultural comparison of patients undergoing ACL reconstruction in the United States and Norway. Knee Surg Sports Traumatol Arthrosc 18: 98-105, 2010.

2. Bartlett RJ, Clatworthy MG and Nguyen TN: Graft selection in reconstruction of the anterior cruciate ligament. J Bone Joint Surg Br 83: 625-634, 2001.

3. Ibrahim SA, Al-Kussary IM, Al-Misfer AR, Al-Mutairi HQ, Ghafar SA and El Noor TA: Clinical evaluation of arthroscopically assisted anterior cruciate ligament reconstruction: Patellar tendon versus gracilis and semitendinosus autograft. Arthroscopy 21: 412-417, 2005.

4. Keays SL, Bullock-Saxton JE, Keays AC, Newcombe PA and Bullock MI: A 6-year follow-up of the effect of graft site on strength, stability, range of motion, function, and joint degeneration after anterior cruciate ligament reconstruction: Patellar tendon versus semitendinosus and Gracilis tendon graft. Am J Sports Med 35: 729-739, 2007.

5. Maeda A, Shino K, Horibe S, Nakata K and Buccafusca G: Anterior cruciate ligament reconstruction with multistranded autogenous semitendinosus tendon. Am J Sports Med 24 504-509, 1996

6. Park MJ, Lee MC and Seong SC: A comparative study of the healing of tendon autograft and tendon-bone autograft using patellar tendon in rabbits. Int Orthop 25: 35-39, 2001.
7. Hu J, Qu J, Xu D, Zhou J and Lu H: Allograft versus autograft for anterior cruciate ligament reconstruction: An up-to-date meta-analysis of prospective studies. Int Orthop 37: 311-320, 2013.

8. Samuelsson K, Andersson D and Karlsson J: Treatment of anterior cruciate ligament injuries with special reference to graft type and surgical technique: An assessment of randomized controlled trials. Arthroscopy 25: 1139-1174, 2009.

9. Nakase J, Kitaoka K, Matsumoto K and Tomita K: Facilitated tendon-bone healing by local delivery of recombinant hepatocyte growth factor in rabbits. Arthroscopy 26: 84-90, 2010.

10. Soon MY, Hassan A, Hui JH, Goh JC and Lee EH: An analysis of soft tissue allograft anterior cruciate ligament reconstruction in a rabbit model: A short-term study of the use of mesenchymal stem cells to enhance tendon osteointegration. Am J Sports Med 35: 962-971, 2007.

11. Atesok K, Fu FH, Wolf MR, Ochi M, Jazrawi LM, Doral MN, Lubowitz JH and Rodeo SA: Augmentation of tendon-to-bone healing. J Bone Joint Surg Am 96: 513-521, 2014.

12. Pan W, Wei Y, Zhou L and Li D: Comparative in vivo study of injectable biomaterials combined with BMP for enhancing tendon graft osteointegration for anterior cruciate ligament reconstruction. J Orthop Res 29: 1015-1021, 2011.

13. Siggia S: The chemistry of polyvinylpyrrolidone-iodine. J Am Pharm Assoc Am Pharm Assoc 46: 201-204, 1957.

14. Balin AK and Pratt L: Dilute povidone-iodine solutions inhibit human skin fibroblast growth. Dermatol Surg 28: 210-214, 2002.

15. von Keudell A, Canseco JA and Gomoll AH: Deleterious effects of diluted povidone-iodine on articular cartilage. J Arthroplasty 28: 918-921, 2013.

16. Schmidlin PR, Imfeld T, Sahrmann P, Tchouboukov A and Weber FE: Effect of short-time povidone-iodine application on osteoblast proliferation and differentiation. Open Dent J 3: 208-212, 2009.

17. Jiang Y, Chen L, Zhang S, Tong T, Zhang W, Liu W, Xu G, Tuan RS, Heng BC, Crawford R, et al: Incorporation of bioactive polyvinylpyrrolidone-iodine within bilayered collagen scaffolds enhances the differentiation and subchondral osteogenesis of mesenchymal stem cells. Acta Biomater 9: 8089-8098, 2013.

18. Gregory CA, Gunn WG, Peister A and Prockop DJ: An Alizarin red-based assay of mineralization by adherent cells in culture: Comparison with cetylpyridinium chloride extraction. Anal Biochem 329: 77-84, 2004.

19. Li H, Ge Y, Wu Y, Jiang J, Gao K, Zhang P, Wu L and Chen S: Hydroxyapatite coating enhances polyethylene terephthalate artificial ligament graft osseointegration in the bone tunnel. Int Orthop 35: 1561-1567, 2011.

20. Livak KJ and Schmittgen TD: Analysis of relative gene expression data using real-time quantitative PCR and the 2(-Delta Delta C(T)) Method. Methods 25: 402-408, 2001.

21. Chundamala $J$ and Wright JG: The efficacy and risks of using povidone-iodine irrigation to prevent surgical site infection: An evidence-based review. Can J Surg 50: 473-481, 2007.

22. Fournel I, Tiv M, Soulias M, Hua C, Astruc K and Aho Glélé LS: Meta-analysis of intraoperative povidone-iodine application to prevent surgical-site infection. Br J Surg 97: 1603-1613, 2010.

23. Brown NM, Cipriano CA, Moric M, Sporer SM and Della Valle CJ: Dilute betadine lavage before closure for the prevention of acute postoperative deep periprosthetic joint infection. J Arthroplasty 27: 27-30, 2012.

24. Martinek V, Latterman C, Usas A, Abramowitch S, Woo SL, Fu FH and Huard J: Enhancement of tendon-bone integration of anterior cruciate ligament grafts with bone morphogenetic protein-2 gene transfer: A histological and biomechanical study. J Bone Joint Surg Am 84-A: 1123-1131, 2002.

25. Chen CH, Liu HW, Tsai CL, Yu CM, Lin IH and Hsiue GH: Photoencapsulation of bone morphogenetic protein-2 and periosteal progenitor cells improve tendon graft healing in a bone tunnel. Am J Sports Med 36: 461-473, 2008.

26. Jadlowiec JA, Celil AB and Hollinger JO: Bone tissue engineering: Recent advances and promising therapeutic agents. Expert Opin Biol Ther 3: 409-423, 2003.

27. Singh M, Dalal S and Singh K: Osteopontin: At the cross-roads of myocyte survival and myocardial function. Life Sci 118: 1-6, 2014.

28. Ramchandani D and Weber GF: Interactions between osteopontin and vascular endothelial growth factor: Implications for skeletal disorders. Bone 81: 7-15, 2015. 